\title{
Ratio of dynamic moduli and estimation of relaxation times
}

Jianhua Huang and Donald G. Baird

Citation: Journal of Rheology (1978-present) 46, 777 (2002); doi: 10.1122/1.1485278

View online: http://dx.doi.org/10.1122/1.1485278

View Table of Contents: http://scitation.aip.org/content/sor/journal/jor2/46/4?ver=pdfcov

Published by the The Society of Rheology

\section{Re-register for Table of Content Alerts}




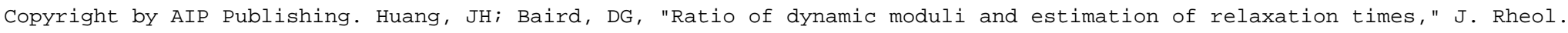
46, 777 (2002); http://dx.doi.org/10.1122/1.1485278

\title{
Ratio of dynamic moduli and estimation of relaxation times
}

\author{
Jianhua Huanga) and Donald G. Baird \\ Department of Chemical Engineering and the Center for Composite Materials \\ and Structures, Virginia Tech, Blacksburg, Virginia 24061-0211
}

(Received 16 April 2001; final revision received 28 March 2002)

\begin{abstract}
Synopsis
In this paper, the theoretical interrelation between the ratio of dynamic moduli and the number and distribution of relaxation times required to fit the generalized Maxwell model to the data was investigated. Theorems were derived for making interval estimation of the relaxation times of the generalized Maxwell model from the ratio of linear combinations of the dynamic moduli at different frequencies. According to these theorems, given dynamic moduli $\left(G^{\prime}\right.$ and $\left.G^{\prime \prime}\right)$ at two frequencies $a$ and $b$ or three frequencies $a, b$, and $(a b)^{1 / 2}$, one must select at least one relaxation time in the relevant interval $\left(\tau_{A}, \tau_{B}\right)$ for the model to fit the data precisely. As a result, from a set of dynamic data $G^{\prime}$ and $G^{\prime \prime}$, one can determine a series of $\left(\tau_{A}, \tau_{B}\right)$ from which the minimum number $\left(N_{\min }\right)$ and distribution (interval estimation of $\tau_{i}$ ) of relaxation times of the model can be estimated. The approach was applied to polystyrene data reported in the literature. The results were discussed and compared with those of relevant work, especially the nonlinear regression model-fitting procedure. (C) 2002 The Society of Rheology. [DOI: 10.1122/1.1485278]
\end{abstract}

\section{INTRODUCTION}

Calculation of the relaxation time spectrum from viscoelastic data and an appropriate viscoelastic model has been a subject of rheological interest for a long time [see, for example, the monograph by Ferry (1980)]. The data and functions often used to determine the relaxation time spectrum are the dynamic moduli. With the generalized Maxwell model, the storage and loss moduli, $G^{\prime}(\omega)$ and $G^{\prime \prime}(\omega)$, may be expressed as functions of the discrete spectrum:

$$
\begin{gathered}
G^{\prime}(\omega)=G_{0}+\sum_{i} G_{i} \omega^{2} \tau_{i}^{2} /\left(1+\omega^{2} \tau_{i}^{2}\right), \\
G^{\prime \prime}(\omega)=\sum_{i} G_{i} \omega \tau_{i} /\left(1+\omega^{2} \tau_{i}^{2}\right),
\end{gathered}
$$

where $G_{0}$ is the equilibrium modulus and $G_{i}$ is the spectral strength associated with a relaxation time $\tau_{i}$. Based on Eqs. (1) and (2) one can find the discrete relaxation time

\footnotetext{
a)Author to whom correspondence should be addressed; electronic mail: jianhua@vt.edu
} 
spectrum by fitting the equations to $G^{\prime}$ and $G^{\prime \prime}$ data. The parameters, $G_{i}$ and $\tau_{i}$, are determined such that the deviation $\left(\mathrm{SD}^{2}\right)$ between predicted values and given $G^{\prime}$ and $G^{\prime \prime}$ data is a minimum:

$$
\mathrm{SD}^{2}=\frac{1}{M} \sum_{k=1}^{M}\left[\left(\frac{G^{\prime}\left(\omega_{k}\right)-G_{N}^{\prime}\left(\omega_{k}\right)}{G^{\prime}\left(\omega_{k}\right)}\right)^{2}+\left(\frac{G^{\prime \prime}\left(\omega_{k}\right)-G_{N}^{\prime \prime}\left(\omega_{k}\right)}{G^{\prime \prime}\left(\omega_{k}\right)}\right)^{2}\right] \rightarrow \text { Min, }
$$

where

$$
G_{N}^{\prime}\left(\omega_{k}\right)=\sum_{i=1}^{N} \frac{\omega_{k}^{2} \tau_{i}^{2}}{1+\omega_{k}^{2} \tau_{i}^{2}} G_{i}+G_{0}, \quad G_{N}^{\prime \prime}\left(\omega_{k}\right)=\sum_{i=1}^{N} \frac{\omega_{k} \tau_{i}}{1+\omega_{k}^{2} \tau_{i}^{2}} G_{i}, \quad G^{\prime}\left(\omega_{k}\right) \quad \text { and } G^{\prime \prime}\left(\omega_{k}\right)
$$

$(k=1,2, \ldots, M)$ are a dataset available from experiments. As shown in Eq. (3), the variables that may influence $\mathrm{SD}^{2}$ and the calculated results include:

(1) $G^{\prime}$ and $G^{\prime \prime}$ data available,

(2) number of the relaxation modes, $N$,

(3) relaxation times $\tau_{i}(i=1,2, \ldots, N)$, and

(4) spectrum strength $G_{i}(i=1,2, \ldots, N)$ and $G_{0}$.

While many computational algorithms have been developed for calculating the discrete relaxation spectrum, all of them can be separated into two categories according to the ways in which $N$ and $\tau_{i}(i=1,2, \ldots, N)$ are determined. In the first approach $N$ and $\tau_{i}(i=1,2, \ldots, N)$ are determined in a somewhat arbitrary way with all times being equally distributed on a logarithmic time scale. Then, a linear regression procedure is used to determine $G_{i}(i=1,2, \ldots, N)$, as was done by Laun (1986). To stabilize the ill posedness of the problem, Honerkamp (1989), Honerkamp and Weese (1989), Elster et al. (1992), and Mead (1994) used different forms of regularization and recovered a model with a large number of modes, $N$. In the second approach $N$ and $\tau_{i}(i$ $=1,2, \ldots N)$, as well as $G_{i}$, are determined by a nonlinear regression procedure. This scheme was first developed by Baumgaertel and Winter (1989) who believed that the number and distribution of the relaxation times could not be preset but had to be optimized through iterative computation. To determine $N$, the algorithms have to iterate from either a large number (say, two per decade), or alternatively from a small number [say, one, as Mustapha and Phillips (2000) proposed] until the minimum number of $N$ that allows a satisfactory fit is obtained. Keeping $N$ small is required to suppress the reproduction of noise in the experimental data and the ill posedness and negative values of $G_{i}$ in the calculated spectrum [Baumgaertel and Winter (1989); Baumgaertel et al. (1990); Winter (1997)].

In the present paper, we will discuss the influence of items (2) and (3) above, i.e., the number and distribution of the relaxation times of the model. More specifically, we attempt to answer the following questions:

Given a set of dynamic data $\left(G^{\prime}\right.$ and $\left.G^{\prime \prime}\right)$, can we estimate the number of relaxation modes $(N)$ and the distribution of $\tau_{i}(i=1,2, \ldots, N)$ in the generalized Maxwell model?

Differing from all regression methods mentioned above, we use analytical relations derived from the ratio of dynamic moduli, not the numerical solution of Eqs. (1) and (2). Our analysis does not include $G_{i}(i=1,2, \ldots, N)$, but is focused on the influence of the relaxation times only. Moreover, instead of dynamic moduli $\left(G^{\prime}, G^{\prime \prime}\right)$ themselves, the ratio of a linear combination of dynamic moduli at different frequencies will be used as a starting point for our analysis. It is found that the ratio of certain functions can reveal some important relations that are difficult to obtain from dynamic moduli themselves. 


\section{RATIOS OF DYNAMIC MODULI AT DIFFERENT FREQUENCIES}

\section{A. Ratios of dynamic moduli for a Maxwell model}

The storage and loss moduli for the Maxwell model, $G_{M}^{\prime}(\omega)$ and $G_{M}^{\prime \prime}(\omega)$, can be expressed, respectively, as

$$
\begin{aligned}
& G_{M}^{\prime}(\omega)=\frac{\omega^{2} \tau^{2}}{1+\omega^{2} \tau^{2}} G, \\
& G_{M}^{\prime \prime}(\omega)=\frac{\omega \tau}{1+\omega^{2} \tau^{2}} G,
\end{aligned}
$$

where $G$ is the elastic modulus associated with $\tau$. We now introduce four functions based on the ratio of linear combination of the dynamic moduli, $G_{M}^{\prime}(\omega)$ and/or $G_{M}^{\prime \prime}(\omega)$ :

$$
\begin{gathered}
f_{1}=f_{1}(a, b, \tau)=\frac{G_{M}^{\prime}(b)}{G_{M}^{\prime}(a)}=\frac{G b^{2} \tau^{2} /\left(1+b^{2} \tau^{2}\right)}{G a^{2} \tau^{2} /\left(1+a^{2} \tau^{2}\right)}=1+\frac{1}{1+b^{2} \tau^{2}}\left(\frac{b^{2}}{a^{2}}-1\right), \\
f_{2}=f_{2}(a, b, \tau)=\frac{G_{M}^{\prime \prime}(b)}{G_{M}^{\prime \prime}(a)}=\frac{G b \tau /\left(1+b^{2} \tau^{2}\right)}{G a \tau /\left(1+a^{2} \tau^{2}\right)}=\frac{a}{b}+\frac{1}{1+b^{2} \tau^{2}}\left(\frac{b}{a}-\frac{a}{b}\right), \\
f_{3}=f_{3}(a, b, \tau)=\frac{G_{M}^{\prime}(b)-G_{M}^{\prime}(a)}{G_{M}^{\prime \prime}(b)+G_{M}^{\prime \prime}(a)}=\frac{m \tau}{1+m^{2} \tau^{2}} \frac{b-a}{m}, \\
f_{4}=f_{4}(a, b, \tau)=\frac{G_{M}^{\prime \prime}(m)}{G_{M}^{\prime \prime}(a)+G_{M}^{\prime \prime}(b)}=\frac{m}{a+b}+\left[\frac{m \tau}{1+m^{2} \tau^{2}} \frac{b-a}{m}\right]^{2} \frac{m}{a+b},
\end{gathered}
$$

where $a$ and $b$ are two arbitrary frequencies and $m=(a b)^{1 / 2}$. To be specific, it is assumed throughout this paper that $a<b$. Note that for constants $a$ and $b$, all of these functions depend on the relaxation time $\tau$ only. Equations (10) and (11) give the derivatives of $f_{1}$ and $f_{2}$ with respect to $\tau$, respectively:

$$
\begin{gathered}
\frac{\partial f_{1}}{\partial \tau}=\frac{2 b^{2}\left(a^{2}-b^{2}\right) \tau}{a^{2}\left(1+b^{2} \tau^{2}\right)^{2}}<0, \\
\frac{\partial f_{2}}{\partial \tau}=\frac{2 b\left(a^{2}-b^{2}\right) \tau}{a\left(1+b^{2} \tau^{2}\right)^{2}}<0 .
\end{gathered}
$$

So, both $f_{1}$ and $f_{2}$ decrease monotonically as $\tau$ increases. From Eqs. (8) and (9) we have

$$
\begin{gathered}
\frac{\partial f_{3}}{\partial \tau}=\frac{(b-a)\left(1-m^{2} \tau^{2}\right)}{\left(1+m^{2} \tau^{2}\right)^{2}}, \\
\frac{\partial f_{4}}{\partial \tau}=\frac{2(b-a) m \tau^{2}}{(b+a)\left(1+m^{2} \tau^{2}\right)^{3}}\left(1-m^{2} \tau^{2}\right) .
\end{gathered}
$$

Clearly, in the region of $\tau<1 / m, \partial f_{3} / \partial \tau>0$, and $\partial f_{4} / \partial \tau>0$; in the region of $\tau$ $>1 / m, \partial f_{3} / \partial \tau<0$ and $\partial f_{4} / \partial \tau<0$. When $\tau=1 / m, \partial f_{3} / \partial \tau=\partial f_{4} / \partial \tau=0, f_{3}$ and $f_{4}$ reach their supremums, $(b-a) /(2 m)$ and $(a+b) /(4 m)$, respectively. The plots of $f_{1}$, $f_{2}, f_{3}$, and $f_{4}$ as a function of $\tau$ are shown in Figs. 1-4, respectively. 


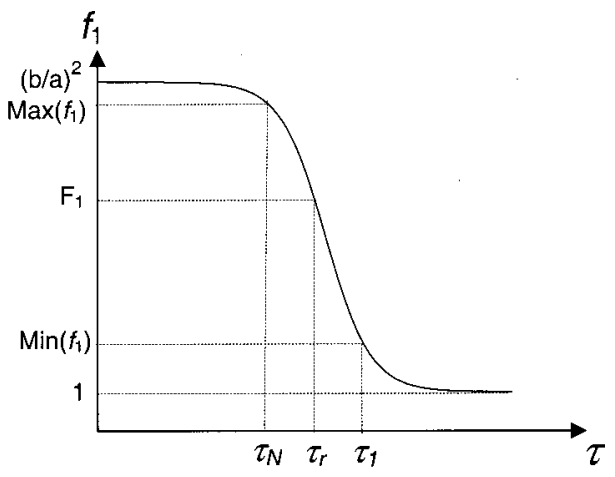

FIG. 1. $f_{1}\left[=G_{M}^{\prime}(b) / G_{M}^{\prime}(a)\right.$, ratio of the storage moduli for a Maxwell element, $\left.a<b\right]$ as a function of $\tau$. $F_{1}$ is the value of the same ratio function for the generalized Maxwell system. $\tau_{r}$ is the $\tau$ coordinate of intersection point of line $f_{1}=F_{1}$ and curve $f_{1}=f_{1}(a, b, \tau) . \tau_{1}$ and $\tau_{N}$ represent the maximum and minimum relaxation times of the system, respectively.

\section{B. Ratios of dynamic moduli for a generalized Maxwell model}

Corresponding to the ratio functions for a single Maxwell model, we define $F_{1}, F_{2}$, $F_{3}$, and $F_{4}$ to represent the four ratio functions for the generalized Maxwell model:

$$
\begin{gathered}
F_{1}=F_{1}(a, b)=\frac{G^{\prime}(b)}{G^{\prime}(a)}, \\
F_{2}=F_{2}(a, b)=\frac{G^{\prime \prime}(b)}{G^{\prime \prime}(a)}, \\
F_{3}=F_{3}(a, b)=\frac{G^{\prime}(b)-G^{\prime}(a)}{G^{\prime \prime}(a)+G^{\prime \prime}(b)},
\end{gathered}
$$

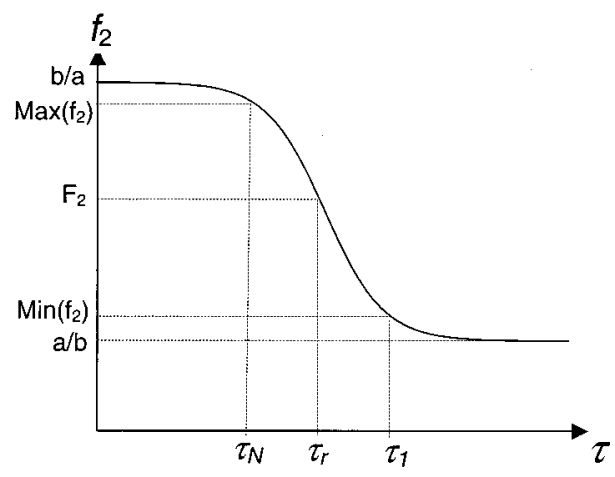

FIG. 2. $f_{2}\left[=G_{M}^{\prime \prime}(b) / G_{M}^{\prime \prime}(a)\right.$, ratio of the loss moduli for a Maxwell element, $\left.a<b\right]$ as a function of $\tau$. $F_{2}$ is the value of the same ratio function for the generalized Maxwell system. $\tau_{r}$ is the $\tau$ coordinate of intersection point of line $f_{2}=F_{2}$ and curve $f_{2}=f_{2}(a, b, \tau) . \tau_{1}$ and $\tau_{N}$ represent the maximum and minimum relaxation times of the system, respectively. 


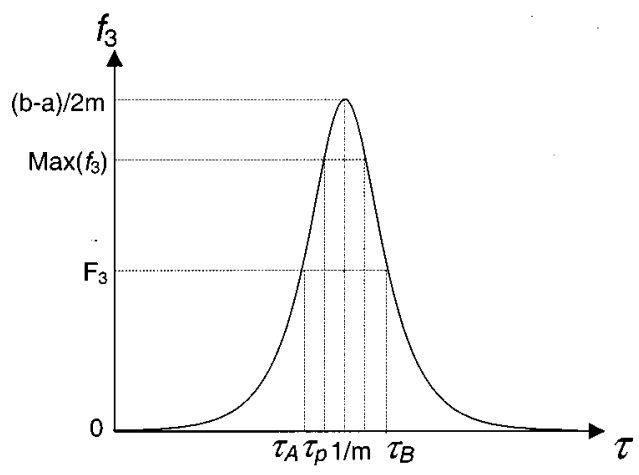

FIG. 3. $f_{3}\left[=\left(G_{M}^{\prime}(b)-G_{M}^{\prime}(a)\right] /\left[G_{M}^{\prime \prime}(b)+G_{M}^{\prime \prime}(a)\right], G_{M}^{\prime}(\omega)\right.$ and $G_{M}^{\prime \prime}(\omega)$ are storage and loss moduli for a Maxwell element, $a<b, m=(a b)^{1 / 2}$ as a function of $\tau$. $F_{3}$ is the value of the same ratio function for the generalized Maxwell system. $\tau_{A}$ and $\tau_{B}$ are $\tau$ coordinates of two intersection points of line $f_{3}=F_{3}$ and curve $f_{3}=f_{3}(a, b, \tau)$, respectively. $\tau_{p}$ is one of the relaxation times of the system corresponding to $f_{3}\left(a, b, \tau_{p}\right)$ $=\operatorname{Max}_{1} \leqslant i \leqslant N\left[f_{3}\left(a, b, \tau_{i}\right)\right]$.

$$
F_{4}=F_{4}(a, b)=\frac{G^{\prime \prime}(m)}{G^{\prime \prime}(a)+G^{\prime \prime}(b)} .
$$

If $G_{i}^{\prime}$ and $G_{i}^{\prime \prime}$ are used to represent the storage and loss moduli for the $i$ th Maxwell element, that is,

$$
\begin{aligned}
& G_{i}^{\prime}(\omega)=\frac{\omega^{2} \tau_{i}^{2}}{1+\omega^{2} \tau_{i}^{2}} G_{i}, \\
& G_{i}^{\prime \prime}(\omega)=\frac{\omega \tau_{i}}{1+\omega^{2} \tau_{i}^{2}} G_{i},
\end{aligned}
$$

then the storage $\left(G^{\prime}\right)$ and loss $\left(G^{\prime \prime}\right)$ moduli for a generalized Maxwell model can be written as

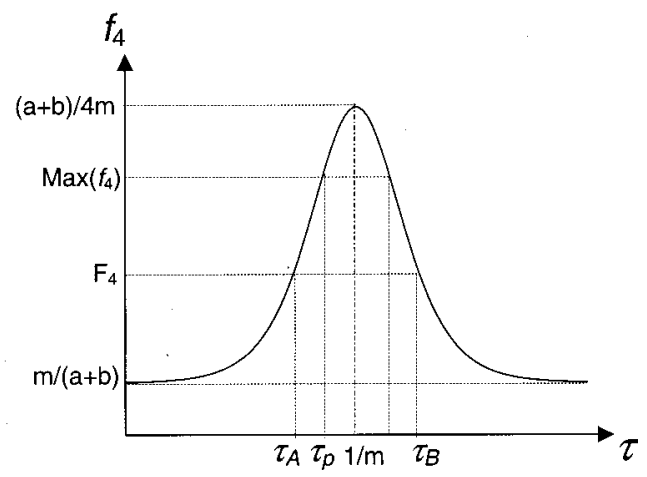

FIG. 4. $f_{4}\left[=G_{M}^{\prime \prime}(m) /\left(G_{M}^{\prime \prime}(b)+G_{M}^{\prime \prime}(a)\right], G_{M}^{\prime \prime}(\omega)\right.$ is loss moduli for a Maxwell element, $a<b, m$ $=(a b)^{1 / 2}$ as a function of $\tau . F_{4}$ is the value of the same ratio function for the generalized Maxwell system. $\tau_{A}$ and $\tau_{B}$ are $\tau$ coordinates of two intersection points of line $f_{4}=F_{4}$ and curve $f_{4}=f_{4}(a, b, \tau)$, respectively. $\tau_{p}$ is one of the relaxation times of the system corresponding to $f_{4}\left(a, b, \tau_{p}\right)=\operatorname{Max}_{1 \leqslant i \leqslant N}\left[f_{4}\left(a, b, \tau_{i}\right)\right]$. 


$$
\begin{gathered}
G^{\prime}(\omega)=G_{0}+\sum_{i=1}^{N} G_{i}^{\prime}(\omega), \\
G^{\prime \prime}(\omega)=\sum_{i=1}^{N} G_{i}^{\prime \prime}(\omega) .
\end{gathered}
$$

If $\tau_{0}$ is associated with an infinite relaxation time, then it follows from Eq. (18) that

$$
G_{0}^{\prime}(\omega)=\frac{\omega^{2} \tau_{0}^{2}}{1+\omega^{2} \tau_{0}^{2}} G_{0}=G_{0}
$$

Thus, Eq. (20) can be rewritten as

$$
G^{\prime}(\omega)=\sum_{i=0}^{N} G_{i}^{\prime}(\omega) .
$$

We have from Eqs. (14) to (17) that

$$
\begin{gathered}
F_{1}(a, b)=\frac{G^{\prime}(b)}{G^{\prime}(a)}=\frac{\sum_{i=0}^{N} G_{i}^{\prime}(b)}{G^{\prime}(a)}=\frac{\sum_{i=0}^{N} f_{1}\left(a, b, \tau_{i}\right) G_{i}^{\prime}(a)}{G^{\prime}(a)}, \\
F_{2}(a, b)=\frac{G^{\prime \prime}(b)}{G^{\prime \prime}(a)}=\frac{\sum_{i=1}^{N} G_{i}^{\prime \prime}(b)}{G^{\prime \prime}(a)}=\frac{\sum_{i=1}^{N} f_{2}\left(a, b, \tau_{i}\right) G_{i}^{\prime \prime}(a)}{G^{\prime \prime}(a)}, \\
F_{3}(a, b)=\frac{G^{\prime}(b)-G^{\prime}(a)}{G^{\prime \prime}(a)+G^{\prime \prime}(b)}=\frac{\sum_{i=1}^{N}\left[G_{i}^{\prime}(b)-G_{i}^{\prime}(a)\right]}{G^{\prime \prime}(a)+G^{\prime \prime}(b)}=\frac{\sum_{i=1}^{N} f_{3}\left(a, b, \tau_{i}\right)\left[G_{i}^{\prime \prime}(a)+G_{i}^{\prime \prime}(b)\right]}{G^{\prime \prime}(a)+G^{\prime \prime}(b)}, \\
F_{4}(a, b)=\frac{G^{\prime \prime}(m)}{G^{\prime \prime}(a)+G^{\prime \prime}(b)}=\frac{\sum_{i=1}^{N} G_{i}^{\prime \prime}(m)}{G^{\prime \prime}(a)+G^{\prime \prime}(b)}=\frac{\sum_{i=1}^{N} f_{4}\left(a, b, \tau_{i}\right)\left[G_{i}^{\prime \prime}(a)+G_{i}^{\prime \prime}(b)\right]}{G^{\prime \prime}(a)+G^{\prime \prime}(b)} .
\end{gathered}
$$

Equations (24)-(27) imply that any ratio function based on the generalized Maxwell model is the average of the functions for the elements with certain weighting functions. As any average value can neither be less than the minimum value nor greater than the maximum value of averaged quantities, the value of the ratio function of the generalized Maxwell model is always between the minimum and maximum values of the function for the elements. That is,

$$
\begin{aligned}
& \operatorname{Min}_{0 \leqslant i \leqslant N}\left[f_{1}\left(a, b, \tau_{i}\right)\right]<F_{1}(a, b)<\underset{0 \leqslant i \leqslant N}{\operatorname{Max}}\left[f_{1}\left(a, b, \tau_{i}\right)\right] \\
& \operatorname{Min}_{1 \leqslant i \leqslant N}\left[f_{2}\left(a, b, \tau_{i}\right)\right]<F_{2}(a, b)<\operatorname{Max}_{1 \leqslant i \leqslant N}\left[f_{2}\left(a, b, \tau_{i}\right)\right], \\
& \operatorname{Min}_{1 \leqslant i \leqslant N}\left[f_{3}\left(a, b, \tau_{i}\right)\right]<F_{3}(a, b)<\operatorname{Max}_{1 \leqslant i \leqslant N}\left[f_{3}\left(a, b, \tau_{i}\right)\right], \\
& \operatorname{Min}_{1 \leqslant i \leqslant N}\left[f_{4}\left(a, b, \tau_{i}\right)\right]<F_{4}(a, b)<\operatorname{Max}_{1 \leqslant i \leqslant N}\left[f_{4}\left(a, b, \tau_{i}\right)\right] .
\end{aligned}
$$

It can be shown that when $G_{0}=0$, Eq. (28) becomes 


$$
\operatorname{Min}_{1 \leqslant i \leqslant N}\left[f_{1}\left(a, b, \tau_{i}\right)\right]<F_{1}(a, b)<\operatorname{Max}_{1 \leqslant i \leqslant N}\left[f_{1}\left(a, b, \tau_{i}\right)\right] .
$$

The proofs of these inequalities are simple and similar. The proof of inequality (30) is given as an example in Appendix A.

\section{RELATIONSHIP BETWEEN THE RATIO OF DYNAMIC MODULI AND THE RELAXATION TIMES}

The functions and relations derived above can be used to correlate the ratios of dynamic moduli with the relaxation times of the generalized Maxwell model. We will now present the theorems for estimating the relaxation times, and discuss briefly how to apply these theorems to dynamic data when experimental errors should be considered. We will also demonstrate the way these theorems are used to estimate the minimum number and distribution of relaxation times when a set of dynamic data is available.

\section{A. Theorems for estimating the relaxation times}

For the generalized Maxwell model with discrete relaxation times, the storage modulus $G^{\prime}$ and loss modulus $G^{\prime \prime}$ can be expressed as

$$
\begin{gathered}
G^{\prime}(\omega)=G_{0}+\sum_{i=1}^{N} G_{i} \omega^{2} \tau_{i}^{2} /\left(1+\omega^{2} \tau_{i}^{2}\right), \\
G^{\prime \prime}(\omega)=\sum_{i=1}^{N} G_{i} \omega \tau_{i} /\left(1+\omega^{2} \tau_{i}^{2}\right) .
\end{gathered}
$$

If $a$ and $b$ represent two arbitrary frequencies $(a<b)$ and $m=(a b)^{1 / 2}$, we have the following relaxation time localization theorems. Note that $\tau_{r}, \tau_{A}$, or $\tau_{B}$ used below do not represent members of $\tau=\left\{\tau_{1}, \tau_{2}, \tau_{3}, \ldots, \tau_{N}\right\}$, which is the collection of the relaxation times for the model.

\section{Theorem 1}

If $\tau=\left\{\tau_{1}, \tau_{2}, \tau_{3}, \ldots, \tau_{N}\right\}\left(\tau_{1}>\tau_{2}>\tau_{3}>\ldots>\tau_{N}\right)$ is the collection of the relaxation times for the generalized Maxwell model whose ratio of dynamic moduli $F_{1}$ $=G^{\prime}(b) / G^{\prime}(a)$, then

$$
\tau_{N}<\tau_{r}
$$

where

$$
\tau_{r}=\sqrt{\frac{1 / a^{2}-F_{1} / b^{2}}{F_{1}-1}} .
$$

If in addition $G_{0}=0$, then

$$
\tau_{1}>\tau_{r}
$$

In other words, the system contains at least one Maxwell element whose relaxation time falls in the interval $\left(0, \tau_{r}\right)$, and at least another one element whose relaxation time falls in $\left(\tau_{r}, \infty\right)$ when $G_{0}=0$. 


\section{Theorem 2}

If $\tau=\left\{\tau_{1}, \tau_{2}, \tau_{3}, \ldots, \tau_{N}\right\}\left(\tau_{1}>\tau_{2}>\tau_{3}>\ldots>\tau_{N}\right)$ is the collection of the relaxation times for the generalized Maxwell model whose ratio of dynamic moduli $F_{2}$ $=G^{\prime \prime}(b) / G^{\prime \prime}(a)$, then

$$
\tau_{N}<\tau_{r}<\tau_{1}
$$

where

$$
\tau_{r}=\sqrt{\frac{b-a F_{2}}{a b\left(b F_{2}-a\right)}} .
$$

In other words, the system contains at least one Maxwell element whose relaxation time falls in the interval $\left(0, \tau_{r}\right)$, and at least one other element whose relaxation time falls in $\left(\tau_{r}, \infty\right)$

\section{Theorem 3}

If $\tau=\left\{\tau_{1}, \tau_{2}, \tau_{3}, \ldots, \tau_{N}\right\}$ is the collection of the relaxation times for the generalized Maxwell model whose ratio of dynamic moduli $F_{3}=\left[G^{\prime}(b)-G^{\prime}(a)\right] /\left[G^{\prime \prime}(a)\right.$ $\left.+G^{\prime \prime}(b)\right]$, then there exists $\tau_{p} \in \tau$ and

$$
\tau_{A}<\tau_{p}<\tau_{B},
$$

where

$$
\begin{gathered}
\tau_{A}=\frac{b-a-\sqrt{(b-a)^{2}-4 a b F_{3}^{2}}}{2 a b F_{3}}, \\
\tau_{B}=\frac{b-a+\sqrt{(b-a)^{2}-4 a b F_{3}^{2}}}{2 a b F_{3}} .
\end{gathered}
$$

In other words, the system contains at least one Maxwell element whose relaxation time falls in the interval $\left(\tau_{A}, \tau_{B}\right)$.

\section{Theorem 4}

If $\tau=\left\{\tau_{1}, \tau_{2}, \tau_{3}, \ldots, \tau_{N}\right\}$ is the collection of the relaxation times for the generalized Maxwell model whose ratio of dynamic moduli $\left.F_{4}=G^{\prime \prime}(m)\right] /\left[G^{\prime \prime}(a)+G^{\prime \prime}(b)\right]$, then there exists $\tau_{p} \in \tau$ and

$$
\tau_{A}<\tau_{p}<\tau_{B},
$$

where

$$
\begin{aligned}
\tau_{A} & =\frac{b-a-\sqrt{(b-a)^{2}-4 a b R^{2}}}{2 a b R}, \\
\tau_{B} & =\frac{b-a+\sqrt{(b-a)^{2}-4 a b R^{2}}}{2 a b R},
\end{aligned}
$$

where 


$$
R=\sqrt{\frac{(a+b) F_{4}}{m}-1} .
$$

In other words, the system contains at least one Maxwell element whose relaxation time falls in the interval $\left(\tau_{A}, \tau_{B}\right)$.

Figures 1-4 are helpful in understanding the meaning of these theorems. In Fig. $1, \tau_{r}$, as defined by Eq. (36), is the $\tau$ coordinate of the intersection point of the line $f_{1}=F_{1}$ and the curve $f_{1}=f_{1}(a, b, \tau)$. It is clear that $\tau_{N}=\operatorname{Min}\left(\tau_{i}\right)<\tau_{r}$ because $f_{1}\left(a, b, \tau_{N}\right)=\operatorname{Max}_{1 \leqslant i \leqslant N}\left[f_{1}\left(a, b, \tau_{i}\right)\right]>F_{1}, \quad$ and $\quad \tau_{1}=\operatorname{Max}\left(\tau_{i}\right)>\tau_{r} \quad$ for $f_{1}\left(a, b, \tau_{1}\right)=\operatorname{Min}_{1 \leqslant i \leqslant N}\left[f_{1}\left(a, b, \tau_{i}\right)\right]<F_{1}$ when $G_{0}=0$. Similarly, theorem 2 can be deduced from Fig. 2 in which $\tau_{r}$ represents the $\tau$ coordinate of the intersection point of the line $f_{2}=F_{2}$ and the curve $f_{2}=f_{2}(a, b, \tau)$. In Fig. 3, $\tau_{A}$ and $\tau_{B}$, as defined by Eqs. (41) and (42), are $\tau$ coordinates of the two intersection points of the line $f_{3}=F_{3}$ and the curve $f_{3}=f_{3}(a, b, \tau)$, and $\tau_{p}$ is one of the relaxation times of the system corresponding to $f_{3}\left(a, b, \tau_{p}\right)=\operatorname{Max}_{1} \leqslant i \leqslant N\left[f_{3}\left(a, b, \tau_{i}\right)\right]$. It is obvious that $\tau_{A}<\tau_{p}$ $<\tau_{B}$ for $f_{3}\left(a, b, \tau_{p}\right)>F_{3}$. The conclusion and logical reasoning of theorem 4 are similar to that of theorem 3 and can also be found from Fig. 4. The complete proof of these theorems can be found in Appendix B of this paper.

\section{B. Estimation of the minimum number of the relaxation times}

The above theorems make it possible to get one interval (the system contains at least one Maxwell element whose relaxation time falls in the interval) by use of dynamic moduli at two $\left(a\right.$ and $b$ ) or three $\left[a, m\right.$, and $b$ with $\left.m=(a b)^{1 / 2}\right]$ frequencies. On this basis, from a set of dynamic moduli $\left(G^{\prime}\right.$ and $G^{\prime \prime}$ at a number of frequencies), one may get a number of such intervals corresponding to different choices of $a$ and $b$. From these intervals one may find a group of intervals that do not overlap with each other, and the number of Maxwell elements involved in the system cannot be smaller than the number of intervals in this group. More specifically, we have the following corollary deduced from the above theorems.

\section{Corollary 1}

Suppose $\left(\tau_{A i}, \tau_{B i}\right)(i=1,2, \ldots)$ is any group of intervals obtained by use of theorems $1-4$ and a set of dynamic moduli $\left(G^{\prime}\right.$ and $G^{\prime \prime}$ at a number of frequencies) of a generalized Maxwell model with the relaxation times $\boldsymbol{\tau}=\left\{\tau_{1}, \tau_{2}, \tau_{3}, \ldots, \tau_{N}\right\}$. If intervals $\left(\tau_{A i}, \tau_{B i}\right)(i=1,2, \ldots, n)$ do not overlap with each other, that is, if

$$
\tau_{A 1}<\tau_{B 1} \leqslant \tau_{A 2}<\tau_{B 2} \leqslant \tau_{A 3}<\tau_{B 3} \cdots \leqslant \tau_{A n}<\tau_{B n},
$$

then $N \geqslant n$.

We note that in the group of intervals of Eq. (47), only interval $\left(\tau_{A 1}, \tau_{B 1}\right)$ and/or $\left(\tau_{A n}, \tau_{B n}\right)$ may be obtained from theorems 1 or 2 . In this case, we define $\tau_{A 1}=0$, $\tau_{B 1}=\tau_{r}$ and $\tau_{A n}=\tau_{r}, \tau_{B n}=\infty$.

\section{Proof of the corollary 1}

According to theorems $1-4$ and Eq. (47), there are $\tau_{p i} \in \boldsymbol{\tau}(i=1,2, \ldots, n)$ and

$$
\tau_{A 1}<\tau_{p 1}<\tau_{B 1} \leqslant \tau_{A 2}<\tau_{p 2}<\tau_{B 2} \leqslant \tau_{A 3}<\tau_{p 3}<\tau_{B 3} \cdots \leqslant \tau_{A n}<\tau_{p n}<\tau_{B n} .
$$

So, the collection $\boldsymbol{\tau}=\left\{\tau_{1}, \tau_{2}, \tau_{3}, \ldots, \tau_{N}\right\}$ contains at least $n$ different members, or $N$ $\geqslant n$. 
Even for the same dynamic dataset, different $n$ may be obtained if the sampling and/or calculation (depending on the theorem and $a, b$ used) methods used are different. The estimation closest to the true value $(N)$ obviously corresponds to the largest $\mathrm{n}$ available, or $n_{\max }$. In this paper we use $N_{\min }$ to represent the minimum number of Maxwell elements involved in the system and let $N_{\min }=n_{\max }$. Because the largest $N_{\min }$ means the best estimation of $N$, and the use of theorems 1-4 comprehensively may lead to the largest possible $n$ and, hence, the best estimation. This is why we introduced different ratios with similar functions, such as $F_{3}$ and $F_{4}$. In Sec. IV A an example will be provided to show how $N_{\min }$ is determined.

\section{Estimating the relaxation times from dynamic data with error}

We now consider the situation when the available $G^{\prime}$ and $G^{\prime \prime}$ data [and, hence, the $\left.F_{x}(x=1,2,3,4)\right]$ contain experimental error. Here, the error tolerance for $F_{x}$ can be determined from the tolerance of the dynamic data $\left(G^{\prime}\right.$ and $\left.G^{\prime \prime}\right)$, which are obtained directly from the experiments. In the following, the tolerance for $F_{x}(x=1,2,3,4)$ is represented by limits $F_{x}^{-}$and $F_{x}^{+}$, or $F_{x}^{-} \leqslant F_{x} \leqslant F_{x}^{+}(x=1,2,3,4)$. Simply substituting $F_{x}^{-}$or $F_{x}^{+}$into Eq. (36) or (39), Eqs. (41) and (42) or Eqs. (44) and (45), one can calculate corresponding relaxation times, $\tau_{r}^{-}, \tau_{r}^{+}, \tau_{A}^{-}$, and $\tau_{B}^{-}$, as well as $\tau_{A}^{+}$and $\tau_{B}^{+}$. The influence of experimental error can be evaluated by comparing these values with those calculated from $F_{x}$. An example here shows how $F_{x}^{-}$and $F_{x}^{+}(x=1,2,3,4)$ are determined from the error tolerance for $G^{\prime}$ and $G^{\prime \prime}$. Let $G^{\prime}(\omega)$ and $G^{\prime \prime}(\omega)$ be the storage and loss modulus, respectively, of a generalized Maxwell model, and let $\hat{G}^{\prime}(\omega)$ and $\hat{G}^{\prime \prime}(\omega)$ be the corresponding observed data. The limit of the experimental error is given by

$$
\begin{aligned}
& \operatorname{Max}_{\omega=a, b, m}\left|\frac{\hat{G}^{\prime}(\omega)-G^{\prime}(\omega)}{G^{\prime}(\omega)}\right| \leqslant \delta_{1}, \\
& \underset{\omega=a, b, m}{\operatorname{Max}}\left|\frac{\hat{G}^{\prime \prime}(\omega)-G^{\prime \prime}(\omega)}{G^{\prime \prime}(\omega)}\right| \leqslant \delta_{2} .
\end{aligned}
$$

From the above equations we have for $\omega=a, b$, and $m$

$$
\begin{aligned}
& \hat{G}^{\prime}(\omega) /\left(1+\delta_{1}\right) \leqslant G^{\prime}(\omega) \leqslant \hat{G}^{\prime}(\omega) /\left(1-\delta_{1}\right), \\
& \hat{G}^{\prime \prime}(\omega) /\left(1+\delta_{2}\right) \leqslant G^{\prime \prime}(\omega) \leqslant \hat{G}^{\prime \prime}(\omega) /\left(1-\delta_{2}\right) .
\end{aligned}
$$

From these equations $F_{x}^{-}$and $F_{x}^{+}(x=1,2,3,4)$ can be defined. For example, from these equations it follows that

$$
\frac{\hat{G}^{\prime \prime}(b)\left(1-\delta_{2}\right)}{\hat{G}^{\prime \prime}(a)\left(1+\delta_{2}\right)} \leqslant \frac{G^{\prime \prime}(b)}{G^{\prime \prime}(a)} \leqslant \frac{\hat{G}^{\prime \prime}(b)\left(1+\delta_{2}\right)}{\hat{G}^{\prime \prime}(a)\left(1-\delta_{2}\right)},
$$

or

$$
F_{2}^{-}=\frac{\hat{G}^{\prime \prime}(b)\left(1-\delta_{2}\right)}{\hat{G}^{\prime \prime}(a)\left(1+\delta_{2}\right)},
$$




$$
F_{2}^{+}=\frac{\hat{G}^{\prime \prime}(b)\left(1+\delta_{2}\right)}{\hat{G}^{\prime \prime}(a)\left(1-\delta_{2}\right)}
$$

\section{APPLICATION OF THE METHOD: ANALYSIS OF POLYSTYRENE MELTS}

\section{A. Calculation of $F_{X}(X=1,2,3,4)$, intervals and $N_{\min }$}

Now we apply the method to polystyrene melts $\left(\right.$ at $\left.180^{\circ} \mathrm{C}\right)$ to show how it works for a given polymeric system. The modulus data were reported in tabulated form (at step of $\log \omega=0.2)$ by Schausberger et al. (1985). The samples denoted as PS-1 to PS-6 are standard polystyrenes with different molecular weights. The detailed molecular parameters are available from the original article. Because the original data have been smoothed and no error tolerance is available from the source, the error or noise in $F_{x}(x$ $=1,2,3,4)$ was not considered here. Because the samples are polymeric melts, it is assumed that $G_{0}=0$ and $F_{1}$ can be used to estimate the maximum relaxation time as indicated by theorem 1. In Table I is given the details of the calculation for PS-5 to illustrate the procedure. The results for all samples are listed in Table II.

\section{Choice of $a, b$ and calculation of $F_{X}$}

Although $G^{\prime}$ and $G^{\prime \prime}$ at any two frequencies $(a$ and $b$ ) can be used to calculate parameters $\tau_{r}$ or $\tau_{A}$ and $\tau_{B}$, the usefulness of the results differs with different choices of $a$ and $b$. Remember our goal is to find the largest number of intervals $\left(\tau_{A i}, \tau_{B i}\right)(i$ $=1,2, \ldots, n)$ without overlap to make the best estimation of $N_{\min }$. To reach this goal one needs to determine intervals with the narrowest length, i.e., the $\left(\tau_{A i}, \tau_{B i}\right)$ with $\left(\tau_{B i} / \tau_{A i}\right) \rightarrow$ min. It was found that $\tau_{A}$ and $\tau_{B}$ calculated from $G^{\prime}$ and $G^{\prime \prime}$ at the nearest frequencies $[(b / a) \rightarrow \mathrm{min}]$ usually have the smallest $\left(\tau_{B} / \tau_{A}\right)$ under the condition that $m\left[=(a b)^{1 / 2}\right]$ is the same. So the neighboring $G^{\prime}$ and $G^{\prime \prime}$ data points were used to calculate $F_{x}$. For example, for PS-5, the $G^{\prime}$ and $G^{\prime \prime}$ data were available from $\log \omega$ $=-3.2$ to +3.8 at a step of 0.2. Starting from $\log \omega=-3.2$ to +3.6 at a step of 0.2 , we choose $\log a=\log \omega$ and $\log b=\log a+0.2$ to calculate $F_{1}=G^{\prime}(b) / G^{\prime}(a), F_{2}$ $=G^{\prime \prime}(b) / G^{\prime \prime}(a)$ and $F_{3}=\left[G^{\prime}(b)-G^{\prime}(a)\right] /\left[G^{\prime \prime}(a)+G^{\prime \prime}(b)\right]$. To calculate $F_{4}$ $=G^{\prime \prime}(m) /\left[G^{\prime \prime}(a)+G^{\prime \prime}(b)\right]$, for $\log \omega=-3.2$ to +3.4 at a step of $0.2, \log a$ $=\log \omega, \log b=\log a+0.4$ and $\log m=\log a+0.2$ are used. The calculated results are listed in Table I. The calculation for other samples (PS-1 to PS-4 and PS-6) is carried out in the same way.

\section{Calculation of $\tau_{r}, \tau_{A}$ and $\tau_{B}$ and $N_{\min }$}

Once $F_{x}(x=1,2,3,4)$ are available, $\tau_{r}$ can be calculated by use of Eq. (36) or (39), whereas $\tau_{A}$ and $\tau_{B}$ can be calculated using Eqs. (41) and (42) or Eqs. (44)-(46). The most convenient way for calculating $F_{x}(x=1,2,3,4)$ and $\tau_{r}$ or $\tau_{A}$ and $\tau_{B}$ is using a spreadsheet such as Microsoft Excel. To calculate $N_{\min }$ from these intervals, a group of intervals without overlap need to be selected. As was discussed in Sec. III B, the selected group should contain as many intervals as possible. The data with block and italic font in Table I show a group of intervals thus determined. The groups for PS-1 to PS-6 are listed in Table II. Note that the way these intervals are determined may not be unique, provided that different ways give rise to the same $N_{\min }$. Also listed in Table II is $N_{\min }$, the minimum number of relaxation modes one needs to select to fit the data. The value of $N_{\min }$ is equal to the number of the intervals in each group, $n$. 
TABLE I. Estimation of the relaxation times for the polystyrene melt (PS-5) at $180{ }^{\circ} \mathrm{C} .{ }^{\mathrm{a}}$

\begin{tabular}{|c|c|c|c|c|c|c|c|c|c|c|c|}
\hline \multicolumn{8}{|c|}{ Theorem 1-3 } & \multicolumn{4}{|c|}{ Theorem 4} \\
\hline $\log b / \log a$ & $F_{1}$ & $\log \tau_{r}$ & $F_{2}$ & $\log \tau_{r}$ & $F_{3}$ & $\log \tau_{A}$ & $\log \tau_{B}$ & $\log b / \log a$ & $F_{4}$ & $\log \tau_{A}$ & $\log \tau_{B}$ \\
\hline $3.8 / 3.6$ & 1.1482 & -3.318 & 1.2589 & -3.942 & 0.1502 & -4.135 & -3.265 & $3.8 / 3.4$ & 0.4914 & -4.055 & -3.145 \\
\hline $3.6 / 3.4$ & 1.1482 & -3.118 & 1.2883 & -3.773 & 0.1664 & -3.875 & -3.125 & $3.6 / 3.2$ & 0.4844 & -3.907 & -2.893 \\
\hline $3.4 / 3.2$ & 1.0715 & -2.748 & 1.2883 & -3.573 & 0.0966 & -3.962 & -2.638 & $3.4 / 3.0$ & 0.4886 & -3.675 & -2.725 \\
\hline $3.2 / 3.0$ & 1.0715 & -2.548 & 1.3183 & -3.406 & 0.1173 & -3.667 & -2.533 & $3.2 / 2.8$ & 0.4815 & -3.530 & -2.470 \\
\hline $3.0 / 2.8$ & 1.0715 & -2.348 & 1.3183 & -3.206 & 0.1443 & -3.358 & -2.442 & $3.0 / 2.6$ & 0.4734 & -3.409 & -2.191 \\
\hline $2.8 / 2.6$ & 1.0233 & -1.897 & 1.2589 & -2.942 & 0.0593 & -3.586 & -1.814 & $2.8 / 2.4$ & 0.4870 & -3.086 & -2.114 \\
\hline $2.6 / 2.4$ & 1.0233 & -1.697 & 1.2589 & -2.742 & 0.0730 & -3.293 & -1.707 & $2.6 / 2.2$ & 0.4783 & -2.959 & -1.841 \\
\hline $2.4 / 2.2$ & 1.0471 & -1.654 & 1.2023 & -2.487 & 0.1739 & -2.646 & -1.954 & $2.4 / 2.0$ & 0.4870 & -2.686 & -1.714 \\
\hline $2.2 / 2.0$ & 1.0233 & -1.297 & 1.1749 & -2.261 & 0.1000 & -2.746 & -1.454 & $2.2 / 1.8$ & 0.4840 & -2.510 & -1.490 \\
\hline $2.0 / 1.8$ & 1.0233 & -1.097 & 1.1220 & -2.013 & 0.1123 & -2.489 & -1.311 & $2.0 / 1.6$ & 0.4866 & -2.290 & -1.310 \\
\hline $1.8 / 1.6$ & 1.0233 & -0.897 & 1.0715 & -1.767 & 0.1205 & -2.253 & -1.147 & $1.8 / 1.4$ & 0.4827 & -2.120 & -1.080 \\
\hline $1.6 / 1.4$ & 1.0471 & -0.854 & 1.0000 & -1.500 & 0.2411 & $\ldots$ & $\ldots$ & $1.6 / 1.2$ & 0.4942 & -1.835 & -0.965 \\
\hline $1.4 / 1.2$ & 1.0233 & -0.497 & 0.9772 & -1.278 & 0.1151 & -1.876 & -0.724 & $1.4 / 1.0$ & 0.4940 & -1.637 & -0.763 \\
\hline $1.2 / 1.0$ & 1.0471 & -0.454 & 0.9550 & -1.056 & 0.2149 & -1.274 & -0.926 & $1.2 / 0.8$ & 0.4875 & -1.483 & -0.517 \\
\hline $1.0 / 0.8$ & 1.0233 & -0.097 & 0.9120 & -0.810 & 0.0968 & -1.561 & -0.239 & $1.0 / 0.6$ & 0.4979 & -1.212 & -0.388 \\
\hline $0.8 / 0.6$ & 1.0471 & -0.054 & 0.9120 & -0.610 & 0.1705 & -1.059 & -0.341 & $0.8 / 0.4$ & 0.4979 & -1.012 & -0.188 \\
\hline $0.6 / 0.4$ & 1.0471 & 0.146 & 0.9120 & -0.410 & 0.1485 & -0.942 & -0.058 & $0.6 / 0.2$ & 0.4979 & -0.812 & 0.012 \\
\hline $0.4 / 0.2$ & 1.0471 & 0.346 & 0.9120 & -0.210 & 0.1294 & -0.817 & 0.217 & $0.4 / 0.0$ & 0.5042 & -0.574 & 0.174 \\
\hline $0.2 / 0.0$ & 1.0471 & 0.546 & 0.9333 & -0.033 & 0.1140 & -0.681 & 0.481 & $0.2 /-0.2$ & 0.4988 & -0.406 & 0.406 \\
\hline $0.0 /-0.2$ & 1.0715 & 0.652 & 0.9333 & 0.167 & 0.1507 & -0.334 & 0.534 & $0.0 /-0.4$ & 0.5050 & -0.169 & 0.569 \\
\hline$-0.2 /-0.4$ & 1.0715 & 0.852 & 0.9550 & 0.344 & 0.1328 & -0.203 & 0.803 & $-0.2 /-0.6$ & 0.4935 & -0.041 & 0.841 \\
\hline$-0.4 /-0.6$ & 1.0965 & 0.983 & 0.9333 & 0.567 & 0.1542 & 0.080 & 0.920 & $-0.4 /-0.8$ & 0.4988 & 0.194 & 1.006 \\
\hline$-0.6 /-0.8$ & 1.0965 & 1.183 & 0.9333 & 0.767 & 0.1313 & 0.191 & 1.209 & $-0.6 /-1.0$ & 0.5050 & 0.431 & 1.169 \\
\hline$-0.8 /-1.0$ & 1.1220 & 1.328 & 0.9550 & 0.944 & 0.1397 & 0.424 & 1.376 & $-0.8 /-1.2$ & 0.4935 & 0.559 & 1.441 \\
\hline$-1.0 /-1.2$ & 1.1749 & 1.442 & 0.9333 & 1.167 & 0.1609 & 0.705 & 1.495 & $-1.0 /-1.4$ & 0.5050 & 0.831 & 1.569 \\
\hline$-1.2 /-1.4$ & 1.2303 & 1.573 & 0.9550 & 1.344 & 0.1626 & 0.911 & 1.689 & $-1.2 /-1.6$ & 0.5115 & 1.068 & 1.732 \\
\hline$-1.4 /-1.6$ & 1.3490 & 1.661 & 1.0000 & 1.500 & 0.1785 & 1.171 & 1.829 & $-1.4 /-1.8$ & 0.5173 & 1.300 & 1.900 \\
\hline$-1.6 /-1.8$ & 1.5488 & 1.722 & 1.0715 & 1.633 & 0.1876 & 1.406 & 1.994 & $-1.6 /-2.0$ & 0.5307 & 1.575 & 2.025 \\
\hline$-1.8 /-2.0$ & 1.7783 & 1.787 & 1.2303 & 1.686 & 0.1709 & 1.542 & 2.258 & $-1.8 /-2.2$ & 0.5116 & 1.669 & 2.331 \\
\hline$-2.0 /-2.2$ & 1.9953 & 1.858 & 1.3804 & 1.718 & 0.1417 & 1.632 & 2.568 & $-2.0 /-2.4$ & 0.4863 & 1.708 & 2.692 \\
\hline$-2.2 /-2.4$ & 2.1380 & 1.958 & 1.4791 & 1.748 & 0.1076 & 1.690 & 2.910 & $-2.2 /-2.6$ & 0.4673 & 1.714 & 3.086 \\
\hline$-2.4 /-2.6$ & 2.2909 & 2.017 & 1.5136 & 1.854 & 0.0795 & 1.747 & 3.253 & $-2.4 /-2.8$ & 0.4631 & 1.843 & 3.357 \\
\hline$-2.6 /-2.8$ & 2.3442 & 2.148 & 1.5488 & 1.897 & 0.0540 & 1.771 & 3.629 & $-2.6 /-3.0$ & 0.4588 & 1.938 & 3.662 \\
\hline$-2.8 /-3.0$ & 2.5119 & $\cdots$ & 1.5849 & $\cdots$ & 0.0378 & 1.813 & 3.987 & $-2.8 /-3.2$ & 0.4513 & $\cdots$ & $\cdots$ \\
\hline$-3.0 /-3.2$ & 2.5119 & $\cdots$ & 1.5849 & $\cdots$ & 0.0238 & 1.811 & 4.389 & & & & \\
\hline
\end{tabular}

${ }^{\mathrm{a}} \mathrm{Unit}$ for all $\tau$ is second. The source data were reported in tabulated form (at step of $\left.\log \omega=0.2\right)$ by Schausberger et al. (1985). From these data $F_{1}=G^{\prime}(b) / G^{\prime}(a), \quad F_{2}=G^{\prime \prime}(b) / G^{\prime \prime}(a), \quad F_{3}=\left[G^{\prime}(b)\right.$ $\left.-G^{\prime}(a)\right] /\left[G^{\prime \prime}(a)+G^{\prime \prime}(b)\right], F_{4}=G^{\prime \prime}(m) /\left[G^{\prime \prime}(a)+G^{\prime \prime}(b)\right]\left[m=(a b)^{1 / 2}\right]$ and corresponding $\tau_{r}$ or $\tau_{A}$ and $\tau_{B}$ are calculated according to theorems $1-4$. From these $\tau_{r}$ and $\left(\tau_{A}, \tau_{B}\right)$ one may find a group of intervals that has the largest members of intervals without overlap, as indicated by the data with block and italic font in the table. These intervals are listed in Table II.

\section{DISCUSSION}

\section{A. Analysis of the results}

Tables I-III show that at the high-frequency end (around $\omega_{\max }$ ), both $F_{1}$ and $F_{2}$ can be used to estimate the shortest relaxation time, $\tau_{N}$, with $F_{2}$ giving a much better prediction. The values of $\tau_{1}$ estimated from $F_{1}$ and $F_{2}$ at the end of low-frequency range are similar except for PS-5. As a whole the $\tau_{1}$ estimated by the present method is close to the $\tau_{1}$ determined by the nonlinear regression method. Considering that our result is actually the lower limit of $\tau_{1}$, it appears that for samples PS- 1 to PS-3, our estimation is 
TABLE II. Relaxation times of polystyrene melts at $180^{\circ} \mathrm{C}$ calculated by the present method (refer to Table I, for example) and nonlinear regression method. ${ }^{\mathrm{a}}$

\begin{tabular}{|c|c|c|c|c|c|c|c|c|}
\hline \multirow[b]{2}{*}{ Sample } & \multicolumn{6}{|c|}{ Present method } & \multicolumn{2}{|c|}{ Nonlinear regression method } \\
\hline & $\log a$ & $\log b$ & $F$ & $\log \tau_{r 1}$ & $\log \tau_{r 2}$ & $N_{\min }$ & $N_{\mathrm{NLR}}$ & $\log \tau_{i}$ \\
\hline \multirow[t]{3}{*}{ PS-1 } & 3.6 & 3.8 & 2 & $-\infty$ & -3.813 & 3 & 3 & -4.590 \\
\hline & 3.2 & 3.6 & 4 & -3.750 & -3.050 & & & -3.292 \\
\hline & 2.0 & 2.4 & 4 & -2.957 & -1.443 & & & -2.807 \\
\hline \multirow[t]{4}{*}{ PS-2 } & 3.6 & 3.8 & 2 & $-\infty$ & -3.861 & 4 & 4 & -4.307 \\
\hline & 3.0 & 3.4 & 4 & -3.662 & -2.738 & & & $-3.517,-2.762$ \\
\hline & 2.2 & 2.4 & 3 & -2.521 & -1.879 & & & -2.097 \\
\hline & 0.8 & 1.2 & 4 & -1.862 & -0.138 & & & \\
\hline \multirow[t]{5}{*}{ PS-3 } & 3.6 & 3.8 & 2 & $-\infty$ & -3.914 & 5 & 6 & -4.186 \\
\hline & 3.0 & 3.4 & 4 & -3.702 & -2.698 & & & -3.382 \\
\hline & 2.0 & 2.4 & 4 & -2.678 & -1.722 & & & $-2.650,-2.089$ \\
\hline & 1.2 & 1.4 & 3 & -1.645 & -0.955 & & & $-1.479,(-0.943)$ \\
\hline & 0.0 & 0.4 & 4 & -0.902 & 0.502 & & & \\
\hline \multirow[t]{7}{*}{ PS-4 } & 3.6 & 3.8 & 2 & $-\infty$ & -3.942 & 7 & 8 & -4.16 \\
\hline & 3.2 & 3.4 & 3 & -3.872 & -2.728 & & & -3.256 \\
\hline & 2.0 & 2.4 & 4 & -2.690 & -1.710 & & & $-2.514,-1.712$ \\
\hline & 1.0 & 1.4 & 4 & -1.602 & -0.798 & & & -0.971 \\
\hline & 0.2 & 0.6 & 4 & -0.766 & -0.034 & & & -0.319 \\
\hline & -0.4 & 0.2 & 3 & 0.018 & 0.582 & & & 0.269 \\
\hline & -1.8 & 1.4 & 4 & 0.738 & 2.462 & & & 0.763 \\
\hline \multirow[t]{8}{*}{ PS-5 } & 3.6 & 3.8 & 2 & $-\infty$ & -3.942 & 8 & 9 & -3.979 \\
\hline & 3.4 & 3.6 & 3 & -3.875 & -3.125 & & & \\
\hline & 2.4 & 2.8 & 4 & -3.086 & -2.114 & & & $(-3.094),-2.223$ \\
\hline & 1.2 & 1.6 & 4 & -1.835 & -0.965 & & & -1.347 \\
\hline & 0.4 & 0.6 & 3 & -0.942 & -0.058 & & & -0.542 \\
\hline & -0.6 & -0.2 & 4 & -0.041 & 0.841 & & & 0.182 \\
\hline & -1.4 & -1.2 & 3 & 0.911 & 1.689 & & & $0.947,1.682$ \\
\hline & -2.4 & -2.0 & 4 & 1.708 & 2.692 & & & 2.225 \\
\hline \multirow[t]{10}{*}{ PS-6 } & 3.6 & 3.8 & 2 & $-\infty$ & -3.989 & 10 & 12 & -4.572 \\
\hline & 2.4 & 3.6 & 3 & -3.981 & -3.019 & & & -3.427 \\
\hline & 2.2 & 2.6 & 4 & -2.927 & -1.873 & & & -2.646 \\
\hline & 1.0 & 1.4 & 4 & -1.700 & -0.699 & & & $(-1.721),-0.889$ \\
\hline & 0.0 & 0.4 & 4 & -0.590 & 0.190 & & & -0.135 \\
\hline & -0.6 & -0.4 & 3 & 0.369 & 0.631 & & & 0.624 \\
\hline & -1.4 & -1.0 & 4 & 0.804 & 1.596 & & & 1.376 \\
\hline & -2.2 & -1.8 & 4 & 1.607 & 2.392 & & & 2.134 \\
\hline & -3.0 & -2.8 & 3 & 2.574 & 3.226 & & & 2.867 \\
\hline & -3.8 & -3.4 & 4 & 3.308 & 3.892 & & & $3.425,3.892$ \\
\hline
\end{tabular}

${ }^{\mathrm{a}}$ Unit for all $\tau$ is second. Nonlinear regression results were cited from Baumgaertel et al. (1990). Data with bracket mean that the relaxation time from nonlinear regression method belongs to no intervals determined by the present method.

more accurate than that obtained by the nonlinear regression, even though the present method is simpler than the regression method. Functions $F_{3}$ and $F_{4}$, on the other hand, are especially useful in determining the intervals $\left(\tau_{A}, \tau_{B}\right)$ between $\tau_{1}$ and $\tau_{N}$. From the percentage of intervals listed in Tables II, $F_{4}$ appears to be more useful than $F_{3}$ in most cases. 
TABLE III. Longest relaxation time $\left(\tau_{1}\right)$ and shortest relaxation time $\left(\tau_{N}\right)$ of polystyrene melts estimated by different methods. ${ }^{\mathrm{a}}$

\begin{tabular}{ccccccc}
\hline \hline Sample & $\begin{array}{c}\text { From } F_{1} \\
\log \tau_{1}>\end{array}$ & $\begin{array}{c}\text { From } F_{2} \\
\log \tau_{1}>\end{array}$ & $\begin{array}{c}\text { From NLR } \\
\log \tau_{1}>\end{array}$ & $\begin{array}{c}\text { From } F_{1} \\
\log \tau_{N}<\end{array}$ & $\begin{array}{c}\text { From } F_{2} \\
\log \tau_{N}<\end{array}$ & $\begin{array}{c}\text { From NLR } \\
\log \tau_{N}\end{array}$ \\
\hline PS-1 & -2.703 & -2.703 & -2.807 & -3.427 & -3.813 & -4.590 \\
PS-2 & -2.052 & -1.903 & -2.097 & -3.394 & -3.861 & -4.186 \\
PS-3 & -0.887 & -0.946 & -0.943 & -3.358 & -3.914 & -4.160 \\
PS-4 & 0.654 & 0.697 & 0.763 & -3.318 & -3.942 & -3.979 \\
PS-5 & 2.148 & 1.897 & 2.225 & -3.318 & -3.942 & -4.572 \\
PS-6 & 3.854 & 3.697 & 3.892 & -3.318 & -3.989 & -4.572 \\
\hline
\end{tabular}

${ }^{a}$ Unit for all $\tau$ is second. Data from NLR (nonlinear regression) method were reported by Baumgaertel et al. (1990) (refer to Table II).

\section{B. Availability of $\tau_{A}$ and $\tau_{B}$ or $\tau_{r}$}

From Table I one can see that for some data groups $\tau_{r}$ or $\tau_{A}$ and $\tau_{B}$ are not available (denoted by $\cdots$ in the table). The reason is that when functions $F_{x}(x=1,2,3,4)$ are substituted into the equations, the items under the square-root sign become negative or the items in the dominator are zero. However, it does not mean that there is anything wrong with the equations. Rather, it tells us that a violation of consistency of the experimental data was detected, which is actually another important purpose of the ratio functions.

\section{Comparison with the results from the nonlinear regression method}

It is interesting to compare our results with the relaxation time distribution determined by nonlinear regression developed first by Baumgaertel and Winter (1989, 1990), who found that the model with a "parsimonious spectrum" (with fewest possible elements) can adequately fit the data $\left(G^{\prime}\right.$ and $\left.G^{\prime \prime}\right)$ without ill posedness as long as the number and distribution of the relaxation times are optimized through iterative computation. Also listed in Table II is the relaxation time distribution determined by Baumgaertel et al. (1990). It can be seen that the $N_{\min }$ determined by the present method is equal or close to the number of relaxation modes determined by the nonlinear regression method $\left(N_{\mathrm{NLR}}\right)$. In addition, generally, one can find one relaxation time in each interval.

\section{Relations between the relaxation times and the frequency window of the given data}

If $\left(\omega_{\min }, \omega_{\max }\right)$ represents the window in which the dynamic moduli are available, one can see from Table II that the values of $\tau_{\min }\left(=\tau_{N}\right)$ predicted by our method are all smaller than $1 / \omega_{\max }$ (PS-5, for example, $\tau_{N} \leqslant 10^{-3.94}$ while $1 / \omega_{\max }=10^{-3.8}$ ), which is in good agreement with $\tau_{N}$ determined by the nonlinear regression method. According to our theorems, any generalized Maxwell model with $\tau_{N} \geqslant 1 / \omega_{\max }$ cannot exactly reproduce the dataset of these samples.

Recently, Davies and Andersen (1997) reported that the interval on which the relaxation spectrum can be precisely determined is the interval 


$$
e^{\pi / 2} / \omega_{\max }<\tau<e^{-\pi / 2} / \omega_{\min }
$$

which is shorter than the reciprocal frequency range $\left(1 / \omega_{\max }, 1 / \omega_{\min }\right)$ over which the data were obtained by 1.36 decades. However, it should be noted that, as was observed by Mustapha and Phillips (2000), the dataset may not be fitted by the model in which the relaxation times are restricted to $e^{\pi / 2} / \omega_{\max }<\tau<e^{-\pi / 2} / \omega_{\min }$. This can now be demonstrated by using the theoretical result presented in this paper. For example, suppose one uses the generalized Maxwell model with $\tau_{N}=e^{\pi / 2} / \omega_{\max }=e^{\pi / 2} / 10^{3.8}=10^{-3.1178}$ to fit the dynamic data of the PS-5 system, then according to Eqs. (7) and (29), one would find that $F_{2}=G^{\prime \prime}\left(\omega=10^{3.8}\right) / \underline{G}^{\prime \prime}(\omega$ $\left.=10^{3.6}\right)<f_{2}\left(a, b, \tau_{N}\right)=0.6705$. Here, $G^{\prime \prime}$ represents the loss modulus of the model. This means that the ratio of $\underline{G}^{\prime \prime}$ reproduced by the generalized Maxwell model with the restriction on $\tau$ as imposed by Davies and Andersen is at best $53.3 \%$ of the experimental value (1.2589, see Table I). Therefore, to allow adequate fit of the data and determination of the relaxation spectrum by either of the two fitting methods (i.e., linear or nonlinear regression), the contribution of relaxation times outside the range $e^{\pi / 2} / \omega_{\max }<\tau<e^{-\pi / 2} / \omega_{\min }$ must be included.

\section{CONCLUSIONS}

The interrelation between the ratio of dynamic moduli at different frequencies and the discrete relaxation times has been found. The ratio functions proposed in this paper can be used to estimate the relaxation times of the generalized Maxwell model or, more specifically, answer the following question:

Given a set of dynamic data $\left(G^{\prime}\right.$ and $\left.G^{\prime \prime}\right)$, can we estimate the number of relaxation modes $(N)$ and the distribution of $\tau_{i}(i=1,2, \ldots, N)$ for the generalized Maxwell model that can adequately fit the data?

The functions $\left(F_{x}, x=1,2,3,4\right)$ proposed are ratios of linear combination of the dynamic moduli $\left(G^{\prime}\right.$ and $\left.G^{\prime \prime}\right)$ at two or three frequencies. Given dynamic moduli $\left(G^{\prime}\right.$ and $\left.G^{\prime \prime}\right)$ at $a$ and $b$, one must select at least one relaxation time in the interval $\left(\tau_{A}, \tau_{B}\right)$ for the model to fit the data precisely. Using the same method, from a set of dynamic data $G^{\prime}$ and $G^{\prime \prime}$ one can determine a series of $\left(\tau_{A}, \tau_{B}\right)$ from which the minimum number $\left(N_{\min }\right)$ and distribution (interval estimation of $\tau_{i}$ ) of the relaxation times of the model can be estimated. The approach was applied to polystyrene data reported in the literature and compared with the nonlinear regression modelfitting method. The $N_{\min }$ thus determined is equal or close to the number of relaxation modes calculated by the nonlinear regression method. The $\left(\tau_{A}, \tau_{B}\right)$ can, in general, predict the relaxation times calculated by nonlinear regression. In particular, the ratio of storage or loss moduli at low frequencies might be used to estimate the longest relaxation time of polymeric fluids easily and accurately. The new functions and method are helpful for determining the discrete relaxation time distribution from dynamic mechanical data. 


\section{APPENDIX A: PROOF OF EQ. (30)}

Note that in Eq. (26),

$$
\begin{aligned}
\sum_{i=1}^{N} f_{3}\left(a, b, \tau_{i}\right)\left[G_{i}^{\prime \prime}(a)+G_{i}^{\prime \prime}(b)\right] & <\sum_{i=1}^{N} \operatorname{Max}_{1 \leqslant j \leqslant N}\left[f_{3}\left(a, b, \tau_{j}\right)\right]\left[G_{i}^{\prime \prime}(a)+G_{i}^{\prime \prime}(b)\right] \\
& =\operatorname{Max}_{1 \leqslant j \leqslant N}\left[f_{3}\left(a, b, \tau_{j}\right)\right] \sum_{i=1}^{N}\left[G_{i}^{\prime \prime}(a)+G_{i}^{\prime \prime}(b)\right] \\
& =\operatorname{Max}_{1 \leqslant j \leqslant N}\left[f_{3}\left(a, b, \tau_{j}\right)\right]\left[G^{\prime \prime}(a)+G^{\prime \prime}(b)\right] .
\end{aligned}
$$

On the other hand,

$$
\begin{aligned}
\sum_{i=1}^{N} f_{3}\left(a, b, \tau_{i}\right)\left[G_{i}^{\prime \prime}(a)+G_{i}^{\prime \prime}(b)\right] & >\sum_{i=1}^{N} \operatorname{Min}_{1 \leqslant j \leqslant N}\left[f_{3}\left(a, b, \tau_{j}\right)\right]\left[G_{i}^{\prime \prime}(a)+G_{i}^{\prime \prime}(b)\right] \\
& =\operatorname{Min}_{1 \leqslant j \leqslant N}\left[f_{3}\left(a, b, \tau_{j}\right)\right] \sum_{i=1}^{N}\left[G_{i}^{\prime \prime}(a)+G_{i}^{\prime \prime}(b)\right] \\
& =\underset{1 \leqslant j \leqslant N}{\operatorname{Min}\left[f_{3}\left(a, b, \tau_{j}\right)\right]\left[G^{\prime \prime}(a)+G^{\prime \prime}(b)\right] .}
\end{aligned}
$$

Applying these inequalities to Eq. (26) yields

$$
\operatorname{Min}_{\leqslant i \leqslant N}\left[f_{3}\left(a, b, \tau_{i}\right)\right]<F_{3}(a, b)<\operatorname{Max}_{1 \leqslant i \leqslant N}\left[f_{3}\left(a, b, \tau_{i}\right)\right],
$$

which is Eq. (30).

\section{APPENDIX B: PROOF OF THEOREMS 1-4}

\section{Proof of theorem 1}

From Eqs. (6) and (28) we have

$$
1+\frac{1}{1+b^{2} \tau_{N}^{2}}\left(\frac{b^{2}}{a^{2}}-1\right)=\operatorname{Max}_{0 \leqslant i \leqslant N}\left[f_{1}\left(a, b, \tau_{i}\right)\right]>F_{1} .
$$

Rearrangement of this inequality gives

$$
\tau_{N}<\sqrt{\frac{1 / a^{2}-F_{1} / b^{2}}{F_{1}-1}}=\tau_{r} .
$$

On the other hand, when $G_{0}=0$, we have from Eq. (32) that

$$
1+\frac{1}{1+b^{2} \tau_{1}^{2}}\left(\frac{b^{2}}{a^{2}}-1\right)=\underset{1 \leqslant i \leqslant N}{\operatorname{Min}}\left[f_{1}\left(a, b, \tau_{i}\right)\right]<F_{1} .
$$

The solution of the inequality is 


$$
\tau_{1}>\sqrt{\frac{1 / a^{2}-F_{1} / b^{2}}{F_{1}-1}}=\tau_{r} .
$$

Thus, theorem 1 is proved.

\section{Proof of theorem 2}

From Eqs. (7) and (29) we have

$$
\frac{a}{b}+\frac{1}{1+b^{2} \tau_{N}^{2}}\left(\begin{array}{ll}
b & \frac{a}{a} \\
\frac{b}{b}
\end{array}\right)=\underset{1 \leqslant i \leqslant N}{\operatorname{Max}}\left[f_{2}\left(a, b, \tau_{i}\right)\right]>F_{2} .
$$

Rearrangement of this inequality gives

$$
\tau_{N}<\sqrt{\frac{b-a F_{2}}{a b\left(b F_{2}-a\right)}}=\tau_{r} .
$$

On the other hand,

$$
\frac{a}{b}+\frac{1}{1+b^{2} \tau_{1}^{2}}\left(\begin{array}{ll}
\frac{b}{a} & -a \\
a
\end{array}\right)=\operatorname{Min}_{1 \leqslant i \leqslant N}\left[f_{2}\left(a, b, \tau_{i}\right)\right]<F_{2} .
$$

The solution of the inequality is

$$
\tau_{1}>\sqrt{\frac{b-a F_{2}}{a b\left(b F_{2}-a\right)}}=\tau_{r} .
$$

So, in conclusion, $\tau_{N}<\tau_{r}<\tau_{1}$.

\section{Proof of theorem 3}

Given $a$ and $b$, one can find a relaxation time $\tau_{p}$ from collection $\tau$ such that

$$
f_{3}\left(a, b, \tau_{p}\right)=\operatorname{Max}_{1 \leqslant i \leqslant N}\left[f_{3}\left(a, b, \tau_{i}\right)\right], \quad \tau_{p} \in \boldsymbol{\tau} .
$$

According to Eqs. (8) and (30) we have

$$
\frac{m \tau_{p}}{1+m^{2} \tau_{p}^{2}} \frac{b-a}{m}=\operatorname{Max}_{1 \leqslant i \leqslant N}\left[f_{3}\left(a, b, \tau_{i}\right)\right]>F_{3} .
$$

Rearrangement of this inequality gives (note that $m^{2}=a b$ )

$$
a b F_{3} \tau_{p}^{2}-(b-a) \tau_{p}+F_{3}<0 .
$$

The solution of the quadratic inequality is

$$
\tau_{A}<\tau_{p}<\tau_{B}
$$

where

$$
\tau_{A}=\frac{b-a-\sqrt{(b-a)^{2}-4 a b F_{3}^{2}}}{2 a b F_{3}}
$$




$$
\tau_{B}=\frac{b-a+\sqrt{(b-a)^{2}-4 a b F_{3}^{2}}}{2 a b F_{3}} .
$$

\section{Proof of theorem 4}

Given $a$ and $b$ one can find a relaxation time $\tau_{p}$ from collection $\boldsymbol{\tau}$ such that

$$
f_{4}\left(a, b, \tau_{p}\right)=\operatorname{Max}_{1 \leqslant i \leqslant N}\left[f_{4}\left(a, b, \tau_{i}\right)\right], \quad \tau_{p} \in \boldsymbol{\tau} .
$$

According to Eqs. (9) and (31) we have

$$
\frac{m}{a+b}+\frac{m}{a+b}\left(\frac{m \tau_{p}}{1+m^{2} \tau_{p}^{2}} \frac{b-a}{m}\right)^{2}=\operatorname{Max}_{1 \leqslant i \leqslant N}\left[f_{4}\left(a, b, \tau_{i}\right)\right]>F_{4} .
$$

Rearrangement of this inequality gives

$$
\frac{m \tau_{p}}{1+m^{2} \tau_{p}^{2}} \frac{b-a}{m}>\sqrt{\frac{(a+b) F_{4}}{m}-1}=R
$$

or

$$
a b R \tau_{p}^{2}-(b-a) \tau_{p}+R<0 .
$$

The solution of the quadratic inequality is

$$
\tau_{A}<\tau_{p}<\tau_{B}
$$

where

$$
\begin{aligned}
& \tau_{A}= \frac{b-a-\sqrt{(b-a)^{2}-4 a b R^{2}}}{2 a b R}, \\
& \tau_{B}=\frac{b-a+\sqrt{(b-a)^{2}-4 a b R^{2}}}{2 a b R},
\end{aligned}
$$

where

$$
R=\sqrt{\frac{(a+b) F_{4}}{m}-1}
$$

\section{References}

Baumgaertel, M. and H. H. Winter, "Determination of discrete relaxation and retardation time spectra from dynamic mechanical data," Rheol. Acta 28, 511-519 (1989).

Baumgaertel, M., A. Schausberger, and H. H. Winter, "The relaxation of polymers with linear flexible chains of uniform length," Rheol. Acta 29, 400-408 (1990).

Davies, A. R. and R. S. Anderssen, "Sampling localization in determining the relaxation spectrum," J. NonNewtonian Fluid Mech. 73, 163-179 (1997).

Elster, J. C., J. Honerkamp, and J. Weese, "Using regulation methods for the determination of relaxation and retardation spectra of polymeric liquids," Rheol. Acta 31, 161-174 (1992).

Ferry, J. D., Viscoelastic Properties of Polymers, 3rd ed. (Wiley, New York, 1980).

Honerkamp, J., "Ill-posed problems in rheology," Rheol. Acta 28, 363-371 (1989). 
Honerkamp, J. and J. Weese, "Determination of the relaxation spectrum by a regularization method," Macromolecules 22, 4372-4377 (1989).

Laun, H. M., "Prediction of elastic strains of polymer melts in shear and elongation," J. Rheol. 30, 459-501 (1986).

Mead, D. W., "Numerical interconversion of linear viscoelastic material functions," J. Rheol. 38, 1769-1795 (1994).

Mustapha, S. M. F. D. S. and T. N. Phillips, "A dynamic nonlinear regression method for the determination of the discrete relaxation spectrum,” J. Phys. D 33, 1219-1229 (2000).

Schausberger, A., G. Schindlauer, and H. Janeschitz-Kriegl, "Linear elasticoviscous properties of molten standard polystyrenes. I. Presentation of complex moduli; role of short-range structural parameters," Rheol. Acta 24, 220-227 (1985).

Winter, H. H., "Analysis of dynamic mechanical data: Inversion into a relaxation time spectrum and consistency check," J. Non-Newtonian Fluid Mech. 68, 225-239 (1997). 